\title{
ANÁLISE DA PRESCRIÇÃO INFORMATIZADA, EM DUAS CLÍNICAS DE UM HOSPITAL UNIVERSITÁRIO
}

\author{
ANALYSIS OF THE COMPUTERIZED PRESCRIPTION IN TWO CLINICS OF A TEACHING HOSPITAL
}

Claudia Câmara Freire ${ }^{1}$; Fernanda Raphael Escobar Gimenes² \& Silvia Helena De Bortoli Cassiani

${ }^{1}$ Enfermeira. Bolsista de Iniciação Científica da FAPESP. ${ }^{2}$ Aluna. Bolsista de Iniciação Científica do CNPq ${ }^{3}$ Docente. Escola de Enfermagem de Ribeirão Preto - USP

Correspondência: Silvia Helena De Bortoli Cassiani. Av. Bandeirantes, 3900 - CEP:14040-902 - Ribeirão Preto-SP E-mail: shbcassi@eerp.usp.br

FREIRE CC; GIMENES FRE \& CASSIANI SHB. Análise da prescrição informatizada, em duas clínicas de um Hospital Universitário. Medicina, Ribeirão Preto, 37: 91-96, jan./jun. 2004.

RESUMO: Objetivo - identificar e analisar a redação da prescrição eletrônica, num Hospital Universitário, do Interior do Estado de São Paulo, e propor sugestões para melhorar o sistema. Modelo do estudo - Estudo avaliatório.

Local do estudo - O estudo foi desenvolvido em duas clínicas do Hospital das Clínicas da Faculdade de Medicina de Ribeirão Preto - USP, utilizando-se, como objeto de análise, as prescrições na forma eletrônica.

População e amostra - Foram analisadas 390 prescrições da Clínica de Ortopedia e 535 prescrições da Clínica Médica, que corresponderam a todas as prescrições avaliadas em 30 dias, não consecutivos.

Resultados - Do total de 925 prescrições avaliadas nas duas clínicas, 112 (12,1\%) apresentaram rasuras, 129 (13,9\%) apresentavam medicamentos suspensos, manualmente ou digitalmente, $261(28,2 \%)$ apresentavam informações que deixavam dúvidas nos profissionais, dentre outros.

Conclusões - As prescrições eletrônicas representam um grande avanço dentro das estratégias utilizadas para minimizar erros decorrentes de prescrições mal formuladas. Entretanto, não erradicam a possibilidade da ocorrência de erros na medicação, fazendo-se necessárias algumas modificações no sistema.

UNITERMOS: Prescrição de Medicamentos. Erros na Medicação. Informática Médica.

\section{1- INTRODUÇÃO E JUSTIFICATIVA}

As prescrições médicas devem conter: nome do paciente, registro, data, nome do medicamento a ser administrado, dosagem, via de administração, freqüência, horário de administração e assinatura do médico. Elas são classificadas em três tipos: prescrições verbais, escritas manualmente e por via eletrônica.

As prescrições eletrônicas, objeto deste estudo, são aquelas nas quais se utiliza o sistema computadorizado, de digitação, seguindo um modelo de dis- posição de dados. Pode-se, também, utilizar um transcritor, para digitar os dados copiados da prescrição redigida manualmente. Esse tipo de prescrição oferece maior segurança, já que elimina dificuldades, na leitura e no entendimento, ocasionadas pela letra ilegível, possibilitando que o erro seja corrigido no momento da digitação, sem que, para isso, haja rasuras ou rabiscos que dificultem ainda mais o entendimento das informações. Assim, as prescrições eletrônicas devem reduzir, significativamente, a freqüência dos sérios erros na medicação ${ }^{(1)}$. 
Há, entretanto, desvantagens, como a possibilidade de ocorrer erros no momento da digitação, sem que o profissional os perceba, como erro nos decimais da dose. Se não houver a disponibilidade de o sistema alertar o médico ou transcritor, no momento da digitação, acerca de possíveis erros, eles podem ser mantidos nas prescrições subseqüentes, quando copiadas da original.

Os sistemas de prescrição eletrônica variam em seu formato, desde sistemas estruturados, que alertam quanto a alergias, interações medicamentosas e doses máximas, até sistemas mais simples, em que a prescrição é simplesmente digitada pelo médico ou transcritor e enviada diretamente ou por fax à farmácia.

No hospital, onde esse estudo foi realizado, a prescrição eletrônica foi introduzida em janeiro de 1998, face à necessidade da melhoria na comunicação entre as áreas de Medicina, Enfermagem e Farmácia. Sua sistematização ocorre da maneira descrita na Figura 1.

Com a introdução desse sistema, houve algumas inovações na realização da prescrição médica, como prescrição digitada pelo médico diretamente no computador; recuperação de qualquer prescrição armazenada no banco de dados, a qualquer momento; prescrição provisória para validação pelo médico docente ou contratado; ausência de transcrição por parte da enfermagem; ficha eletrônica de controle de antimicrobianos; dispensação de medicamentos através de prescrição enviada por via eletrônica; padronização de medicamentos de estoque e utilização do nome genérico.

Os erros devidos à prescrição contribuem significativamente para o índice total de erros de medicação e têm elevado potencial para resultarem em conseqüências maléficas para o paciente. Estima-se que, em cada dez pacientes admitidos no hospital, um esteja em risco potencial ou efetivo, de erro na medicação ${ }^{(2)}$. Esse risco aumenta à medida em que os profissionais não conseguem ler corretamente devido à letra ilegível ou à falta de informações necessárias para a correta administração, como via, freqüência, etc. ${ }^{(3,4)}$

Além da dificuldade da leitura do nome dos medicamentos, a assinatura do médico, se ilegível, também favorece situações complicadas, pois os profissionais não sabem a quem contatar para esclarecer possíveis dúvidas.

Para evitar erros devidos à redação da prescrição médica, estudos apontam medidas que têm sido tomadas, como: padronização de processos, uso da prescrição eletrônica, aprimoramento e ênfase à educação dos profissionais que prescrevem, e expansão da ação dos farmacêuticos clínicos para melhor integração da equipe de saúde. Todavia, é a introdução da prescrição eletrônica uma das tentativas mais sérias e mais efetivas para a prevenção dos erros na medicação ${ }^{(5,6)}$.

Há poucos estudos realizados no Brasil, analisando a prescrição eletrônica, mesmo porque são poucos os hospitais que a estão empregando, dado o custo da implantação e manutenção. Considera-se, todavia, que a implantação desse sistema deva ocorrer, brevemente, em muitos hospitais brasileiros, principalmente nos universitários ou nos de grande porte.

Dada a inovação da implantação do sistema de prescrição eletrônica no hospital em que atuamos, e no Brasil, planejou-se este estudo, com o intuito de proceder a uma análise do sistema em funcionamento atualmente, buscando contribuir tanto para seu aperfeiçoamento como para sua divulgação. A prescrição médica, clara, legível, correta e completa contribui no cuidado de enfermagem de qualidade e na segurança dos pacientes $^{(7,8,9)}$, daí a motivação para a realização deste estudo por profissionais da enfermagem.
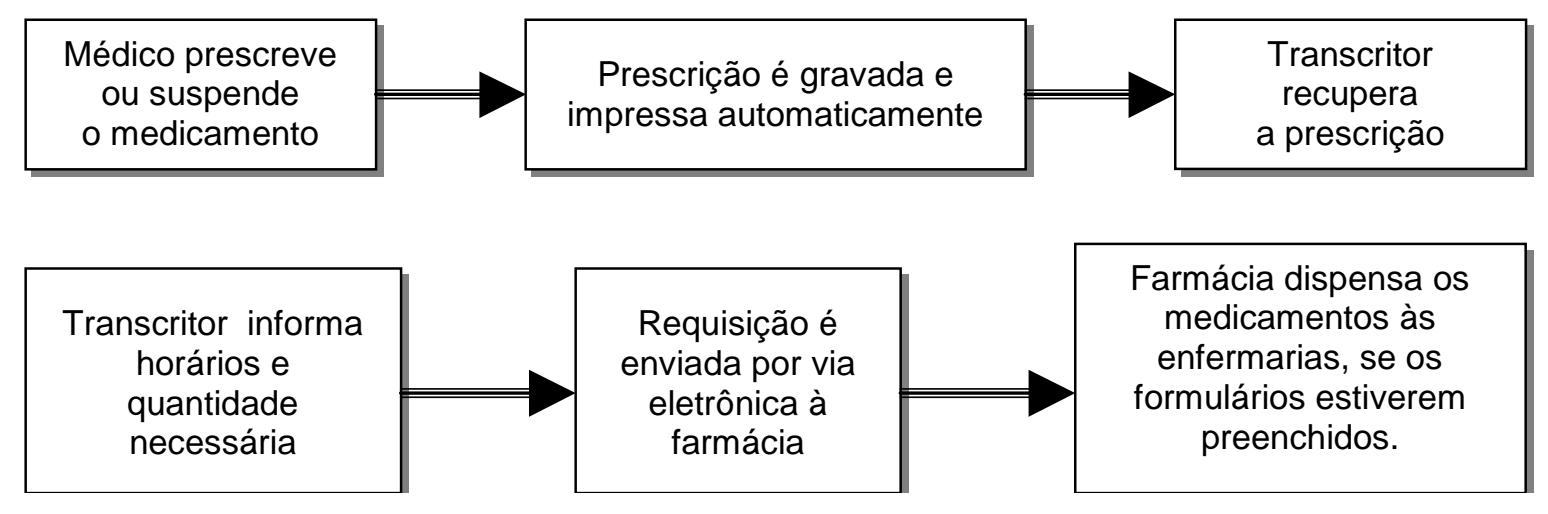

Figura 1 - Processo de distribuição da medicação, a partir da prescrição eletrônica. Ribeirão Preto, 2003 


\section{2- OBJETIVOS}

Este estudo teve por objetivos identificar as falhas na redação da prescrição eletrônica e propor sugestões para a melhoria do sistema, a partir de dados extraídos da literatura.

\section{3- MATERIAL E MÉTODOS}

Tipo de estudo - Trata-se de estudo descritivo do tipo avaliativo, que é uma forma aplicada de pesquisa, que envolve descobrir como um programa está funcionando, prática ou politicamente ${ }^{(10)}$.

Local do estudo - $\mathrm{O}$ estudo foi desenvolvido na Clínica Médica e na Clínica de Ortopedia do Hospital das Clínicas da Faculdade de Medicina de Ribeirão Preto - USP. A seleção dessas clínicas deve-se ao fato de elas receberem pacientes, para quem, na maioria das vezes, há grande número de medicamentos prescritos ou que necessitem de longa estada no hospital devido a sua patologia.

População e Amostra - Foram analisadas 390 prescrições da Clínica de Ortopedia e 535 prescrições da Clínica Médica, que corresponderam a todas as prescrições computadorizadas, realizadas em 30 dias não consecutivos, perfazendo um total de 925 prescrições.
Procedimento de Coleta de Dados - A coleta dos dados teve início após a aprovação do projeto pelo Comitê de Ética em Pesquisa do referido hospital. Foi utilizado um formulário com itens relacionados à análise da redação da prescrição, como: presença de rasuras, medicamentos suspensos, prescrições escritas manualmente, informações não claras, que poderiam deixar dúvidas nos profissionais da enfermagem, no momento de preparação e administração, com informações sobre nome, dose, via de administração e sobre procedimentos de administração dos medicamentos.

A coleta dos dados foi realizada perto do posto de enfermagem e no momento em que todas as prescrições do dia já tivessem sido realizadas.

\section{4- RESULTADOS E DISCUSSÃO}

A Figura 2 sintetiza os resultados apresentados depois da análise dos dados de cada clínica.

Observa-se que, do total de prescrições analisadas na Clínica Médica (535), 17\% (91) apresentaram rasuras, ou seja, riscos ou círculos nos nomes, ou na dose, na frequiência e no número de dias. Na Clínica Ortopédica, das 390 prescrições analisadas, 5,4\% (21) delas apresentaram-se rasuradas. Supõe-se que, devido à maior demanda de prescrições e às freqüentes

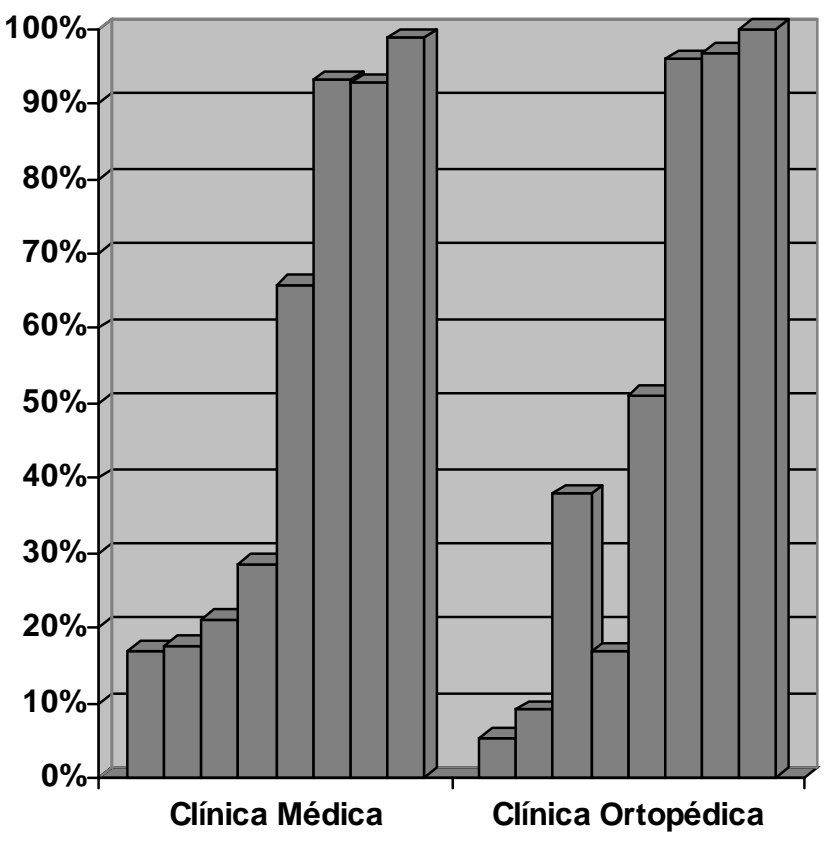

$\square$ Presença de rasuras
$\square$ Presença de medicamentos
suspensos
$\square$ Presença de informações
duvidosas
$\square$ Medicamentos prescritos à mão
$\square$ Presença de dose de apresentação
e dose prescrita
$\square$ Informações sobre a via de
administração
$\square$ Presença de horário
$\square$ Informações sobre procedimentos
de administração

Figura 2 - Distribuição, por clínica, dos dados analisados nas prescrições. Ribeirão Preto, 2003. 
mudanças de estado dos pacientes, na Clínica Médica, ocorram mais alterações nas prescrições. Assim sendo, muitos medicamentos são suspensos e outros prescritos, constantemente.

É preciso atentar para os riscos decorrentes dessa prática, pois a presença de rasuras pode causar confusão na leitura, resultando em erros e possíveis danos ao paciente. Observa-se, ainda, que, no $\mathrm{HC}$, os profissionais de enfermagem seguem a cópia da prescrição, emitida pela farmácia, quando da dispensação de medicamentos, para administrá-los e, portanto, alterações na prescrição original precisam ser comunicadas à equipe, pelo risco de serem administrados medicamentos anteriormente prescritos e agora suspensos ou serem omitidos medicamentos prescritos, manualmente, após a impressão da prescrição eletrônica.

Quanto à presença de medicamentos suspensos, percebemos que, na Clínica Médica, 17,6\% (94) das prescrições analisadas continham medicamentos suspensos, sendo que $96,8 \%$ deles foram suspensos, manualmente, pelo médico. Na Ortopedia, 9\%(35) das prescrições analisadas continham medicamentos suspensos, sendo que, em todas, os medicamentos foram suspensos pelo método manual.

Considera-se o potencial que essa prática tem sobre os erros de medicação, já que, muitas vezes, a equipe de enfermagem não é comunicada sobre a suspensão do medicamento ${ }^{(11,12)}$.

Verificamos, também, a presença de informações que poderiam deixar dúvidas nos profissionais da enfermagem, tais como o uso de abreviações, ausência de dados sobre a via de administração e dose de apresentação ou informações necessárias, mas não presentes. Observou-se que a Clínica Ortopédica apresentou $37,9 \%$, e a Clínica Médica, 21,1\%. Essas informações incluíam abreviaturas, como, por exemplo, "BIC", "ACM", "PMV", "pos: 1 med."; medicamentos prescritos manualmente e não legíveis; frases incompletas, que não dão informações claras (dar em dias alternados); e, também, o uso de decimais ("100.00 ml").

Constatou-se, ainda, que 16,7\% (65) das prescrições, na Clínica Ortopédica, continham o nome dos medicamentos escritos manualmente, enquanto que, na Clínica Médica, em 28,6\%(153) das prescrições, muitos dos nomes estavam escritos de forma ilegível. Tal atitude pode contribuir para aumentar as chances de erro, visto que a prescrição ilegível pode gerar dúvidas nos profissionais.

As prescrições devem conter a dose de apresentação do medicamento, para que ele seja solicitado à farmácia, e, também a dose prescrita, para que a equipe de enfermagem saiba a quantidade exata que se deve administrar no paciente. Assim, constatamos que, das 535 prescrições analisadas, na Clínica Médica, 65,9\%(352) continham dose de apresentação e dose prescrita. Das 390 prescrições analisadas na Ortopedia, 51\%(198) continham dose de apresentação e dose prescrita.

Esses resultados indicam a necessidade da conscientização dos profissionais, uma vez que há, ainda, ausência de informações necessárias nas prescrições, tais como a dose correta da administração.

Em ambas as clínicas, não houve erros na redação de quaisquer dos medicamentos prescritos por via eletrônica. Isso se justifica pelo fato de que os nomes dos medicamentos utilizados são padronizados pela instituição e gravados no sistema informatizado, portanto o médico só necessita de digitar as iniciais do nome do medicamento e, automaticamente, o nome aparece inteiro na tela do computador.

Não houve erros na redação dos medicamentos escritos manualmente, que estavam legíveis, e nem nos nomes de medicamentos digitados que estavam fora do estoque da farmácia e, portanto, não eram padronizados.

95,9\%(374) das prescrições, continham, informações sobre a via de administração do medicamento, na Clínica Ortopédica, enquanto, na Clínica Médica, esse valor foi de 93,1\% (498), sendo que, em ambas as clínicas, os medicamentos digitados por via eletrônica, foram os que apresentaram maior participação nesses resultados.

Vale destacar que houve prescrições em que a informação sobre a via de administração não aparecia; assim, supõe-se que foi necessário que o profissional de enfermagem tirasse sua dúvida com a enfermeira, ou com o médico ou através da prescrição anterior. Nessas situações, o medicamento poderia ter sido administrado em uma via inadequada, podendo gerar graves danos ao paciente e ao profissional.

Quanto à presença do horário da administração ou freqüência, na Clínica Médica, 92,7\%(495) das prescrições continham uma ou outra e, na Clínica Ortopédica, o valor foi de 96,7\% (377). Embora a maioria das prescrições apresentassem informações sobre o horário de administração ou frequiência, o ideal seria que todas elas tivessem a informação.

Todas as prescrições (100\%) da Clínica Ortopédica continham informações sobre procedimentos de administração dos medicamentos. Na Clínica Médica, a porcentagem de prescrições que continham essas informações foi de $98,7 \%$ (528) havendo, en- 
tão, uma porcentagem de 1,3\% de prescrições cujo procedimento de administração de algum medicamento não era mencionado.

\section{5- CONCLUSÃO}

Os dados obtidos neste estudo indicam que, embora a prescrição seja informatizada, há, ainda, prescrições rasuradas, com medicamentos suspensos e escritos de forma manual. Além disso, muitas prescrições apresentaram informações que deixavam dúvidas naqueles que preparavam e administravam o medicamento, além de, em outras, haver ausência de informações, como dose de apresentação e dose prescrita, horário e via de administração.

A falta de clareza na prescrição pode confundir o profissional, podendo causar possíveis danos ao paciente. Estudos referem que os erros adversos das medicações são caros para os sistemas de saúde, podendo resultar em sérias conseqüências ${ }^{(12,13)}$, além de elevar o tempo de internação. Os custos diretamente ligados aos erros de medicação variam de $\$ 1900$ a $\$ 5900$ dólares por paciente ${ }^{(14)}$.

Estudo realizado, nos Estados Unidos da América, também demonstrou que a dose de apresentação das drogas, redigida inadequadamente é um tipo de erro bastante comum nas prescrições ${ }^{(4,13)}$.

Observando os problemas identificados neste estudo e comparando-os com os dados da literatura, apresentam-se, a seguir, os elementos que a National Coordinating Council for Medication Error Reporting and Prevention (NCCMERP) dos Estados Unidos da América recomenda ${ }^{(15)}$ :

- Todas as prescrições devem ser legíveis, e os médicos devem utilizar um sistema de entrada da prescrição diretamente pelo computador.

- As prescrições devem conter uma breve anotação do propósito, se o médico considerá-la apropriada.

- Todas as prescrições devem ser escritas em sistema métrico, exceto nas terapias que usam procedimentos padronizados, tais como insulina, vitaminas, etc. As unidades devem ser escritas por extenso ao invés de serem abreviadas ("U").

- Deve ser incluída a idade e, quando apropriado, o peso do paciente.

- A prescrição deve incluir o nome do medicamento, a dose e a concentração.

- O zero deve sempre preceder às expressões numéricas abaixo de um, porém o zero terminal não deve nunca ser usado após décimos.

- O uso de abreviaturas deve ser evitado e instru- ções vagas como: "tomar como indicado" ou "quando necessário" devem ser evitadas.

Embora o sistema computadorizado de prescrições represente um grande avanço dentro das estratégias utilizadas para minimizar erros decorrentes de prescrições mal formuladas, esse sistema, da forma como utilizado, não erradicou a possibilidade de erros na medicação, necessitando, portanto, de algumas modificações, como a de alertar os médicos quanto à dose exacerbada, histórico de alergia do paciente, freqüência inadequada e interações medicamentosas; impossibilitar a digitação e uso de abreviações e de símbolos; conscientizar os profissionais médicos quanto à importância dos programas de treinamento para utilização do sistema; requisição do medicamento diretamente da farmácia, no momento em que a prescrição está sendo feita; impossibilitar a redação manual de medicamentos e não identificar doses com zeros que possam confundir a enfermagem, como, por exemplo, 1000,00 $\mathrm{ml}^{(3,9,11,16)}$.

A implementação de um sistema mais elaborado, com apoio à decisão clínica, concomitantemente com melhor educação por parte daqueles que prescrevem, tornará possível a elaboração de prescrições mais detalhadas e fáceis de serem compreendidas pelos profissionais que as manuseiam. Assim, os eventos adversos ao paciente poderão ser reduzidos a partir do sucesso desse programa, melhorando a qualidade do cuidado.

Este estudo analisou a redação das prescrições eletrônicas, em duas clínicas de uma instituição hospitalar, e os aspectos que podem afetar a preparação e administração da medicação e conseqüentemente a segurança do paciente. Embora existam vários estudos americanos, ${ }^{(16,17)}$ analisando a tecnologia da informação nos hospitais, há poucos no Brasil e, portanto, divulgar experiências é necessário. Evidenciou-se, entretanto, que há práticas que coexistem com a prescrição eletrônica, que têm impacto negativo na segurança do processo de medicação e necessitam de modificação.

Enquanto avaliação do sistema através da análise da redação das prescrições, este estudo mostrou um dos aspectos, entretanto, outros estudos são necessários, que analisem o custo dessas práticas, comparação com outras clínicas, e análise a partir de maior amostra de prescrições.

Ressalta-se que a prescrição eletrônica ainda é um projeto caro e complexo e, portanto, poucas são as instituições que contam com esse sistema, mesmo nos EUA ${ }^{(18)}$. Reconhece-se que muitas instituições podem estar discutindo e planejando a introdução desse sistema e, assim, este estudo poderá contribuir no sentido de difundir as experiências já existentes. 
FREIRE CC; GIMENES FRE \& CASSIANI SHB. Analysis of the computerized prescription in two clinics of a teaching hospital. Medicina, Ribeirão Preto, 37: 91-96, jan/june 2004.

ABSTRACT: Objective: To identify and analyze the writing of electronic orders in a university hospital in São Paulo State and propose suggestions to improve the system.

Study model: Evaluative study.

Study site: The study was conducted in two clinics at the University Hospital of the Faculty of Medicine of Ribeirão Preto - University of São Paulo - and its object of analysis consisted of electronic orders.

Population and sample: 390 orders were analyzed in the orthopedics clinic and 535 orders were evaluated in the medical clinic, which corresponded to all orders evaluated in 30 nonconsecutive days.

Results: Of the total number of 925 orders evaluated in the two clinics, $112(12.1 \%)$ presented erasures, $129(13.9 \%)$ showed medication suspensions introduced either manually or in typing, $261(28.2 \%)$ presented information that made professionals doubtful, among others.

Conclusions: Electronic orders represent a great advancement in the strategies used to minimize errors resulting from badly formulated orders. However, it does not eradicate the possibility of occurrence of medication errors; which indicates that modifications in the system are necessary.

UNITERMS: Prescriptions, Drug. Medication Errors. Medical Informatics.

\section{REFERÊNCIAS BIBLIOGRÁFICAS}

1 - KAUSHAL R. Medication errors and adverse drug events in pediatric inpatients. JAMA 285: 2114-2120, 2001.

2 - ZANGWILL AB. Reducing prescribing errors through a quiz program for medical residents. Am J Health - Syst Pharm 57:1396-1397, 2000.

3 - WINSLOW EH; NESTOR VA; DAVODOFF SK; THOMPSON $P G$ \& BORUM JC. Legibility and completeness physician handwritten medication order. Heart \& Lung 26: 158-164, 1997.

4 - LESAR TS. Factors related to errors in medication prescribing. JAMA 277: 312-317, 1997.

5 - BATES DW. Improving medication safety across institutions. J Qual Impr 26: 319-320, 2000.

6 - HATFIELD G. Rating hospitals for medication safety: a scorecard. Pharm Times 65: 42-45, 1999.

7 - ARGO A; COX KK \& KELLY WN. The ten most common lethal medication errors in hospital patients. Hosp Pharm 35: 470-474, 2000.

8 - GANDHI TK; SEGER D \& BATES DW. Identifying drug safety issues : From researches to practice. Int $\mathbf{J}$ Qual Health Care 12: 69-76, 2000.

9 - LEAPE L. System analisys of adverse drug events. JAMA 274: 35-43, 1995.

10 - POLIT DF \& HUNGLER BP. Nursing research principles and methods. $7^{\text {th }}$.ed. , J.B. Lippincott CO, Philadelphia,1999.

11 - DEAN B; SCHACHTER M; VINCENT C \& BARBER N. Causes of prescribing errors in hospital inpatients: a prospective study. LANCET 359: 1373-1378, 2002.
12 - BATES DW; LEAPE LL; CULLEN DJ; LAIRD N; PETERSEN LA; TEICH JM; BURDICK EB; HICKEY M; KLEEFIELD S; SHEA B; VLIET MV \& SEGER DL. Effect of computerized physician order entry and a team intervention on prevention of serious medication errors. JAMA 280: 1311-1316, 1998.

13 - KAUSHAL R; BARKER KN \& BATES DW. How can information technology improve patient safety and reduce medication errors in children's health care? Arch Pediatr Adolesc Med 155: 1002-1006, 2001.

14 - RASCHKE RA; GOLLIHARE B; WUNDERLICH TA; GUIDRY JR; LEIBOWITZ AI; PEIRCE JC; LEMELSON L; HEISLER MA \& SUSONG C. A computer alert system to prevent injury from adverse drug events. JAMA 280: 1317-1320, 1998.

15 - COUSINS DM. Practitiones experienses as a catalyst for change : The USP medication errors reporting program. In: ESCOVITZ A; PATHAK DS \& SCHNEIDER PJ, eds. Improving the quality of the medication use process. Pharmaceutical Products Press, New York, Cap 6, p.71-96, 1998.

16 - BATES DW. Incidence of adverse drug event and potential adverse drug events: implications for prevention. JAMA 274: 29-34, 1995.

17 - ZARINS K. Computer-based prescribing. Nurs Times 90: 56-59, 1994.

18 - KUPERMAN G \& GIBSON RF. Computer physician order entry: benefits, costs, and issues. Ann Intern Med 139: 31-39, 2003.

Recebido para publicação em 12/08/2003

Aprovado para publicação em 29/12/2003 